\title{
Energetic and Kinetic Contributions of Contact Residues of Antibody D1.3 in the Interaction with Lysozyme
}

\author{
Patrick England, François Brégégère, and Hugues Bedouelle* \\ Protein Engineering Group (CNRS-URA 1129), Unité de Biochimie Cellulaire, Institut Pasteur, \\ 28, rue du Docteur Roux, 75724 Paris Cedex 15, France
}

Received June 13, 1996; Revised Manuscript Received September 4, $1996^{\otimes}$

\begin{abstract}
Fully functional variable fragments (Fv) of D1.3, a mouse antibody directed against the hen egg lysozyme, were readily produced as hybrids (Fv-MalE) with the maltose-binding protein of Escherichia coli and purified independently of their antigen-binding properties. We used site-directed mutations of residues in the complementarity-determining regions (CDRs) of D1.3 as local conformational probes, and compared their effects on the binding of Fv and Fv-MalE to lysozyme. We found that the MalE moiety did not significantly interfere with the interaction between the antigen and the antibody Fv fragment. We then determined the contribution of several potential contact residues of D1.3 in the interaction with lysozyme, by assaying the effect of site-directed mutations on the kinetics of association and dissociation of the complex between Fv-MalE and immobilized lysozyme, using the BIAcore apparatus. While the $k_{\text {on }}$ values were virtually unaffected by the mutations, the $k_{\text {off }}$ values varied by more than three orders of magnitude. Both charged (aspartate and arginine) and aromatic (tyrosine and tryptophan) residues in the CDR3 regions of the heavy and light chains of D1.3, which form the center of its antigen-combining site, played a preponderant part in the binding of lysozyme. Our results also showed that indirect hydrogen bonds, bridged by water molecules, contributed significantly to the interaction between D1.3 and lysozyme, and that their energy could be estimated at 1 to $2 \mathrm{kcal} \cdot \mathrm{mol}^{-1}$.
\end{abstract}

A large number of high-resolution crystallographic studies have lead to a better understanding of the molecular details of protein-protein interfaces (Janin \& Chothia, 1990). Potential contacts between the partners can be defined with great precision by such methods, but structural analysis cannot show by itself which interactions are energetically important for tight binding nor how protein-protein docking kinetically occurs. Comprehensive studies of the part played by each contact atom in the association and the dissociation processes are fundamental to understand the chemical basis of macromolecular recognition and should, in turn, open up the pathway for rational protein and drug design.

Interaction between antibodies and protein antigens is an excellent model for studying protein-protein interaction (Webster et al., 1994; Braden \& Poljak, 1995; Davies \& Cohen, 1996). One of the best-characterized complexes is that between the hen egg white lysozyme (HEL) ${ }^{1}$ and the mouse monoclonal antibody D1.3. Three-dimensional structures of the heterodimeric variable fragment $(\mathrm{Fv})$ in both its liganded and unliganded states have been determined by X-ray crystallography at $1.8 \AA$ resolution (Bhat et al., 1994). These data suggest that residues from all six complementa-

* Corresponding author. Tel: +33 14568 8379. FAX: +3314061 3043. E-mail: hbedouel@pasteur.fr.

${ }^{\otimes}$ Abstract published in Advance ACS Abstracts, December 1, 1996.

${ }^{1}$ Abbreviations: CDR, complementarity-determining region; Fv, variable fragment; HEL, hen egg lysozyme; IPTG, isopropyl $\beta$-Dthiogalactoside; $k_{\text {off }}$, dissociation rate constant; $k_{\mathrm{on}}$, association rate constant; $K_{\mathrm{d}}^{\prime}$, equilibrium dissociation constant at the solid-liquid interface; mAb, monoclonal antibody; MalE, maltose-binding protein of Escherichia coli; RIA, radioimmunoassay; RU, resonance units; SPR, surface plasmon resonance. Mutants are named with the prefix denoting the heavy chain $(\mathrm{H})$ or the light chain $(\mathrm{L})$ variable domain, followed by the single-letter code for the residue found in the wild-type antibody (D1.3), then the sequence position, and finally the residue in the mutant. rity-determining regions (CDR) of D1.3 contact HEL, through as many as 20 hydrogen bonds and 44 Van der Waals contacts. Several indirect hydrogen bonds, bridged by water molecules, could also help stabilize the complex between the antibody and the antigen.

Three studies in which contact residues of D1.3 were mutated to determine their contribution in the energy of binding to HEL, have been reported. However, two of them only measured the effects of multiple mutations, so preventing a clear appraisal of the role of individual contact residues (Ito et al., 1993, 1995). The other one lacked kinetic data related to the association and dissociation processes. It also failed to establish the part played by two seemingly important residues, either because the production of the corresponding mutant antibodies was too low or because they could not be purified on a column of immobilized HEL (Hawkins et al., 1993).

Here we used the technique of surface plasmon resonance (SPR), as implemented on the BIAcore apparatus (Pharmacia Biosensor) (Malmqvist, 1993), to measure the effect of single mutations in D1.3 on its kinetics of binding to HEL. Ten mutations, involving contact residues from all six CDRs of the antibody, were constructed. We expressed the mutant Fv fragments of D1.3 as fusion proteins with the maltosebinding protein of Escherichia coli (MalE). These Fv-MalE hybrids were purified using MalE as an affinity handle, independently of their properties of binding to HEL (Brégégère et al., 1994).

The use of several other proteins as fusion partners with antibody fragments has been reported, such as enzymes (Neuberger et al., 1984; Wels et al., 1992; Goshorn et al., 1993; Newton et al., 1996), toxins (Brinkmann \& Pastan, 
pVD 82

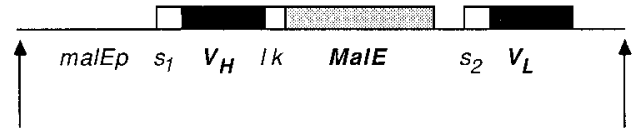

pVD 91

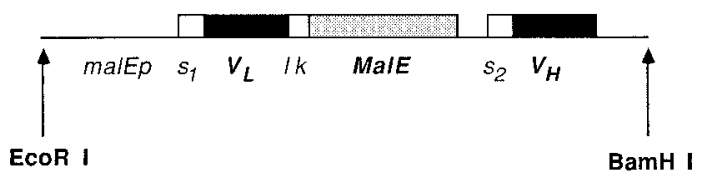

FIGURE 1: Structures of the Fv-MalE coding regions of phagemids pVD82 and pVD91. The two phagemids coded for heterodimers $\mathrm{V}_{\mathrm{L}}:: \mathrm{V}_{\mathrm{H}}-$ MalE and $\mathrm{V}_{\mathrm{H}}:: \mathrm{V}_{\mathrm{L}}-$ MalE respectively, where the symbol $(::)$ represents a non-covalent assembly and the symbol $(-)$ represents a covalent linkage between the $\mathrm{C}$-terminal end of the variable domain and the $\mathrm{N}$-terminal end of mature MalE. The dicistronic operons encoding the Fv-MalE hybrids were under the control of the malEp promoter. They were carried by an EcoRI$B a m \mathrm{HI}$ restriction fragment and inserted between the corresponding restriction sites of phagemid pTZ18R (Mead et al., 1986). $V_{H}$ and $V_{L}$ code for the $\mathrm{V}_{\mathrm{H}}$ and $\mathrm{V}_{\mathrm{L}}$ domains of antibody D1.3, while malE codes for the mature protein MalE, $l k$ for the linker (N-Ala-AspAla-Ala-Ala-C), $s 1$ for the MalE signal peptide, and $s 2$ for the PhoA signal peptide.

1994), or binding proteins (Dübel et al., 1995; Neri et al., 1995), illustrating the versatility of such constructions. However, it has yet to be shown that these fusion partners do not interfere with the interaction between the antigen and the antibody fragment. To address this question, we resorted to a functional approach, in which site-directed mutations of residues of D1.3, distributed all over the paratope, are used as local conformational probes: their effect on the binding of hybrid Fv-MalE to HEL was compared with that reported in the case of free Fv (i.e., not fused with MalE) (Hawkins et al., 1993).

\section{MATERIALS AND METHODS}

(A) Expression Vectors for the Fv-MalE Hybrids. Phagemids pVD81 and pVD91 have been described (Brégégère et al., 1994). We used phagemid pVD82, a derivative of pVD81, and pVD91 to express hybrids between the wildtype Fv fragment of D1.3 and MalE (Figure 1). We derived pVD82 from pVD81 by site-directed mutagenesis, using the oligonucleotide 5'-CTCTCACAGTGAGCTCAGCCGATGCTGCAGCTAAAATCGAAGAAGGT-3'. The mutagenesis replaced the linker peptide between $\mathrm{V}_{\mathrm{H}}$ and MalE $(\mathrm{N}$ Ala-Lys-Thr-Thr-Pro-Pro-Ala-C) by a shorter peptide (NAla-Asp-Ala-Ala-Ala-C, coded by the underlined sequence), and introduced a unique $\mathrm{SacI}$ restriction site. We introduced mutations into the Fv-MalE hybrids by oligonucleotide sitedirected mutagenesis of pVD91 and pVD82. The mutageneses were performed as described, with the single-stranded DNAs of the phagemids as templates (Kunkel et al., 1987). The sequences of the mutated genes (either $\mathrm{V}_{\mathrm{H}}$ or $\mathrm{V}_{\mathrm{L}}$ ) were checked by the dideoxy chain-termination method, using the T7-Sequencing Kit (Pharmacia Biotech).

(B) Production and purification of the Fv-MalE hybrids. To produce the Fv-MalE hybrids from phagemids pVD82, pVD91 and their mutants, we used PD28 (recA, DmalE444, malT ${ }^{c}$; Duplay et al., 1987) as a host strain, and LB medium, containing $200 \mu \mathrm{g} / \mathrm{mL}$ ampicillin and the indicated supplements, as a culture medium. The PD28 derivatives were grown overnight at $30{ }^{\circ} \mathrm{C}$, in the presence of $10 \mathrm{mg} / \mathrm{mL}$ glucose to repress the malE promoter. The cells were collected by centrifugation, resuspended in fresh medium (4

times the initial volume), and then incubated for $6 \mathrm{~h}$ at 22 ${ }^{\circ} \mathrm{C}$ in the presence of $1 \mathrm{mg} / \mathrm{mL}$ glucose and $0.2 \mathrm{mM}$ IPTG. The periplasmic extracts were prepared as described (Bedouelle \& Duplay, 1988). Full-length hybrids were purified by affinity chromatography on a column of cross-linked amylose followed by ion-exchange chromatography on a FPLC Mono-Q HR5/5 column (Pharmacia) (Brégégère et al., 1994). The purity of the hybrids was checked by electrophoresis through SDS-polyacrylamide gels and staining with Coomassie Brilliant Blue. Their concentration was measured by the Bio-Rad protein assay. They were stocked at $4{ }^{\circ} \mathrm{C}$ in $30 \mathrm{mM}$ Tris- $\mathrm{HCl}, \mathrm{pH} 7.5,50 \mathrm{mM} \mathrm{NaCl}, 1 \mathrm{mM}$ maltose.

(C) Determination of the Reactivity of Fv-MalE toward HEL. mAb56.5, a monoclonal antibody directed against MalE, was covalently immobilized on the carboxymethylated dextran surface of a CM5 sensorchip to a level of 70008000 resonance units (RU), using the Amine Coupling Kit (Pharmacia Biosensor). The resulting derivatized surface, CM5(mAb56.5), was equilibrated with buffer $\mathrm{A}$ at a temperature of $20{ }^{\circ} \mathrm{C}$ and a flow rate of $5 \mu \mathrm{L} / \mathrm{min}$, conditions which were used in all the subsequent steps. Buffer A was $10 \mathrm{mM}$ phosphate buffer, $\mathrm{pH} 7.4,2.7 \mathrm{mM} \mathrm{KCl}, 137 \mathrm{mM}$ $\mathrm{NaCl}, 0.005 \%$ detergent P20 (Pharmacia), and $1 \mathrm{mM}$ maltose. The addition of maltose prevents any dimerization of MalE (Richarme, 1982).

In a preliminary experiment, a solution of wild-type or mutant Fv-MalE was injected in parallel onto CM5(mAb56.5) (Figure 2: curve "Fv-MalE alone") and onto an underivatized CM5 surface (not shown). The association was followed between times $t_{0}$ and $t_{1}$, and the dissociation was followed between times $t_{1}$ and $t_{2} . \Delta R(\mathrm{Fv}-\mathrm{MalE})$, the increment in the SPR signal due to the specific capture of Fv-MalE by CM5(mAb56.5), was the difference between the two measured SPR responses. In a second experiment, FvMalE was injected onto CM5(mAb56.5) between $t_{0}$ and $t_{1}$ as above, and then a saturating solution of HEL (Merck, 1.5 $\mu \mathrm{M}$ or more) was injected onto the complex Fv-MalE·CM5(mAb56.5) between times $t_{1}$ and $t_{2}$ (Figure 2: curve "FvMalE + HEL"). A background signal was determined in a control experiment by injecting HEL alone across CM5(mAb56.5) between $t_{1}$ and $t_{2}$, without prior capture of FvMalE (Figure 2: curve "HEL alone"). $\Delta R(\mathrm{HEL})$, the increment in the SPR signal due to the specific capture of HEL by Fv-MalE·CM5(mAb56.5) was obtained by substracting $\Delta R(\mathrm{Fv}-\mathrm{MalE})$ (determined in the preliminary experiment) and the background signal (measured in the control experiment) from the SPR signal measured in the second experiment.

The reactivity was calculated as the ratio

$$
[\Delta R(\mathrm{HEL}) / M(\mathrm{HEL})] /[\Delta R(\mathrm{Fv}-\mathrm{MalE}) / M(\mathrm{Fv}-\mathrm{MalE})]
$$

where $M(\mathrm{HEL})$ and $M(\mathrm{Fv}-\mathrm{MalE})$ are respectively the molecular masses of HEL (14 400) and of Fv-MalE (66 200), using the fact that SPR responses, measured in RU, are proportional to the surface concentration of bound protein (a variation of $1 \mathrm{kRU}$ corresponds to a change of about 1 $\mathrm{ng} \cdot \mathrm{mm}^{-2}$; Stenberg et al., 1991).

(D) Determination of the Reactivity of Immobilized HEL toward Fv-MalE. HEL was covalently immobilized on CM5 sensorchips to a level $\Delta R(\mathrm{imHEL})=700-900 \mathrm{RU}$, using the Amine Coupling Kit. A sample of wild-type Fv-MalE 


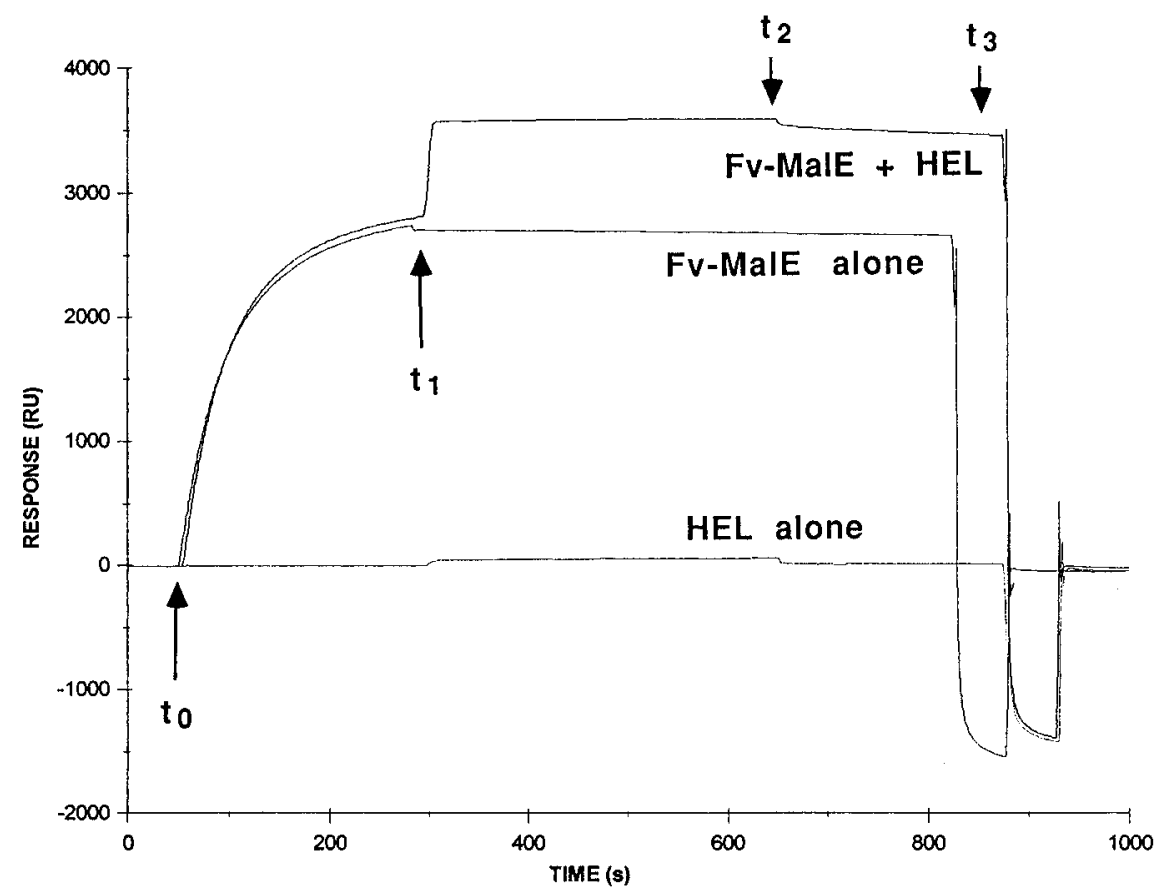

FIGURE 2: Determination of the reactivity of a Fv-MalE hybrid toward HEL, using the BIAcore apparatus. Fv-MalE + HEL: A solution of Fv-MalE $(20 \mu \mathrm{L})$ was injected between times $t_{0}$ and $t_{1}$ onto CM5(mAb56.5), a surface derivatized with a monoclonal antibody directed against MalE. At time $t_{1}$, a saturating solution of HEL $(30 \mu \mathrm{L} ; 1.5 \mu \mathrm{M}$ in this particular experiment) was injected onto the Fv-MalE. CM5(mAb56.5) complex, yielding a global SPR response of 3597 RU. The injection ended at time $t_{2}$, after which some dissociation, mainly of the HEL·Fv-MalE complex, could be observed. At time $t_{3}$, the surface was regenerated by injection of $5 \mu \mathrm{L}$ of $50 \mathrm{mM} \mathrm{HCl}$. Fv-MalE alone: Fv-MalE was injected between $t_{0}$ and $t_{1}$, without subsequent injection of HEL, yielding a SPR response of $2741 \mathrm{RU}$. HEL alone: HEL was injected between $t_{1}$ and $t_{2}$ without previous injection of Fv-MalE, yielding a response of 54 RU. The background signal obtained when injecting Fv-MalE alone onto an underivatized CM5 surface was -135 RU (not shown). The values of the specific increments in SPR signals were calculated as specified in Materials and Methods: $\Delta R(\mathrm{Fv}-\mathrm{MalE})=2741-(-135)=2876 \mathrm{RU}$, and $\Delta R(\mathrm{HEL})=3597-2876-54=667 \mathrm{RU}$. Therefore, in this specific example, the reactivity, calculated according to eq 1 was as shown below.

$$
[\Delta R(\mathrm{HEL}) / \mathrm{M}(\mathrm{HEL})] /[\Delta R(\mathrm{Fv}-\mathrm{MalE}) / \mathrm{M}(\mathrm{Fv}-\mathrm{MalE})]=(667 / 14400) /(2876 / 66200)=1.07
$$

was injected in parallel onto the CM5(HEL) surface and onto an underivatized CM5 surface at a saturating concentration $(1 \mu \mathrm{M})$, in the conditions described in part $\mathrm{C}) . \Delta R(\mathrm{Fv}-$ MalE), the increment in SPR signal due to the capture of Fv-MalE by CM5(HEL), was the difference between the two measured SPR responses. The fraction of immobilized HEL molecules that were available for Fv-MalE binding, was calculated as the ratio

$$
[\Delta R(\mathrm{Fv}-\mathrm{MalE}) / M(\mathrm{Fv}-\mathrm{MalE})] /[\Delta R(\mathrm{imHEL}) / M(\mathrm{HEL})]
$$

(E) Binding Assays. The molecular interactions between the Fv-MalE hybrids and immobilized HEL were measured with the BIAcore apparatus, in buffer $\mathrm{A}$, at $20^{\circ} \mathrm{C}$ and a flow rate of $5 \mu \mathrm{L} / \mathrm{min}$. The samples of hybrids $(20-30 \mu \mathrm{L})$ were injected across the CM5(HEL) surface at concentrations ranging from $1 \mathrm{nM}$ to $10 \mu \mathrm{M}$. Ten to 15 different concentrations were used for each wild-type or mutant hybrid. The dissociation was followed for $5 \mathrm{~min}$. We observed no significant difference in the kinetics of dissociation whether free HEL was present or not, at a saturating concentration of $1.5 \mu \mathrm{M}$ or more, as a competitive ligand for Fv-MalE. This observation showed that no rebinding of Fv-MalE to CM5(HEL) occurred during the dissociation phase in the conditions used. At the end of each experiment, the HEL surface was regenerated by an injection of $5 \mu \mathrm{L}$ of $50 \mathrm{mM} \mathrm{HCl}$.
(F) Evaluation of the Kinetic Data. The kinetics of interaction between the Fv-MalE hybrids and the immobilized HEL were followed with the BIAcore apparatus as described above. The recorded data were then analyzed with a nonlinear least-squares algorithm implemented in the BIAevaluation 2.1 software package (Pharmacia Biosensor), as described (O'Shannessy et al., 1993). Goodness of fitting to the kinetic data was evaluated by $\chi^{2}$ tests.

In the first instance, we attempted to fit simple exponential functions to the experimental data. We used

$$
R(t)=\left(R_{\mathrm{d} 0}-R_{\mathrm{d} \infty}\right) \mathrm{e}^{-k_{\mathrm{off}}\left(t-t_{\mathrm{d} 0}\right)}+R_{\mathrm{d} \infty}
$$

for the dissociation profiles, where $R(t), R_{\mathrm{d} 0}$, and $R_{\mathrm{d} \infty}$ are respectively the values of the SPR responses (in RU) at the times $t, t_{\mathrm{d} 0}$ (start of measures), and $t_{\mathrm{d} \infty}$ (after complete dissociation of the complex between FV-MalE and HEL). $k_{\text {off }}$ and $R_{\mathrm{d} \infty}$ are fitting parameters. Similarly, we used

$$
R(t)=\left(R_{\mathrm{a} \infty}-R_{\mathrm{a} 0}\right)\left(1-\mathrm{e}^{-k_{\mathrm{obs}}\left(t-t_{\mathrm{a} 0}\right)}\right)+R_{\mathrm{a} 0}
$$

for the association profiles, where $R_{\mathrm{a} 0}$ and $R_{\mathrm{a} \infty}$ are respectively the SPR responses at times $t_{\mathrm{a} 0}$ (start of measures) and $t_{\mathrm{a} \infty}$ (when the formation of the complex between FV-MalE and HEL has reached steady-state). $k_{\mathrm{obs}}, R_{\mathrm{a} 0}$, and $R_{\mathrm{a} \infty}$ are fitting parameters.

If both the populations of immobilized HEL and of circulating Fv-MalE are homogeneous, and if their interaction 
is reversible and non-cooperative, then $k_{\text {off }}$ is the dissociation rate constant of the complex and $k_{\mathrm{obs}}$ the apparent association rate constant. $k_{\text {off }}$ does not vary as a function of $\mathrm{C}$, the molar concentration of Fv-MalE, and we used hereafter the mean of the values that we determined for each concentration. In contrast, $k_{\mathrm{obs}}$ follows the linear relation

$$
k_{\mathrm{obs}}=k_{\mathrm{on}} C+k_{\mathrm{off}}
$$

where $k_{\text {on }}$ is the real association rate constant of the complex. We henceforth determined $k_{\text {on }}$ as the slope of the secondary plot of $k_{\mathrm{obs}}$ against $C . K_{\mathrm{d}}^{\prime}$, the equilibrium dissociation constant of the complex between Fv-MalE and immobilized HEL, measured at the solid-liquid interface, is obtained by the ratio

$$
K_{\mathrm{d}}^{\prime}=k_{\mathrm{off}} / k_{\mathrm{on}}
$$

We also analyzed the dissociation and the association profiles as sums of two exponential functions of time:

$$
\begin{gathered}
R(t)=\left(R_{\mathrm{d} 0}-R_{\mathrm{d} 1}\right) \mathrm{e}^{-k_{\mathrm{off} 1}\left(t-t_{\mathrm{d} 0}\right)}+ \\
\left(R_{\mathrm{d} 1}-R_{\mathrm{d} \infty}\right) \mathrm{e}^{-k_{\mathrm{off} 2}\left(t-t_{\mathrm{d} 0}\right)}+R_{\mathrm{d} \infty} \\
R(t)=\left(R_{\mathrm{a} 1}-R_{\mathrm{a} 0}\right)\left(1-\mathrm{e}^{-k_{\mathrm{obs} 1}\left(t-t_{\mathrm{a} 0}\right)}\right)+ \\
\left(R_{\mathrm{a} \infty}-R_{\mathrm{a} 1}\right)\left(1-\mathrm{e}^{-k_{\mathrm{obs} 2}\left(t-t_{\mathrm{a} 0}\right)}\right)+R_{\mathrm{a} 0}
\end{gathered}
$$

In the case of reversible and non-cooperative interactions between a homogeneous population of circulating Fv-MalE and two distinct populations of immobilized HEL, yielding two different complexes, $k_{\text {off1 }}$ and $k_{\text {off2 }}$ on the one hand and $k_{\mathrm{obs} 1}$ and $k_{\mathrm{obs} 2}$ on the other hand represent partial rate constants. $k_{\text {off1 }}$ and $k_{\text {off2 }}$ do not vary as a function of $C$, and we henceforth used the mean of the values that we determined for each concentration. $k_{\mathrm{obs} 1}$ and $k_{\mathrm{obs} 2}$ follow linear relations similar to eq 5. Two partial association rate constants $\left(k_{\mathrm{on} 1}\right.$ and $\left.k_{\mathrm{on} 2}\right)$ and two equilibrium dissociation constants $\left(K_{\mathrm{d} 1}^{\prime}\right.$ and $\left.K_{\mathrm{d} 2}^{\prime}\right)$ can therefore be determined as described above. The initial rate of association is given by

$$
\mathrm{d} R / \mathrm{d} t\left(t=t_{\mathrm{a} 0}\right)=R_{\mathrm{eq} 1} k_{\mathrm{obs} 1}+R_{\mathrm{eq} 2} k_{\mathrm{obs} 2}
$$

where $R_{\mathrm{eq} 1}=R_{\mathrm{a} 1}-R_{\mathrm{a} 0}$ and $R_{\mathrm{eq} 2}=R_{\mathrm{a} \infty}-R_{\mathrm{a} 1}$ are the contributions of the two exponential functions to the overall amplitude of the reaction.

\section{RESULTS}

(A) Expression and Purification of the Fv-MalE Hybrids. We used phagemids pVD82 and pVD91 to express respectively hybrids $\mathrm{V}_{\mathrm{L}}:: \mathrm{V}_{\mathrm{H}}-$ MalE and $\mathrm{V}_{\mathrm{H}}:: \mathrm{V}_{\mathrm{L}}-\mathrm{MalE}$, in which one of the variable domains $\left(\mathrm{V}_{\mathrm{H}}\right.$ or $\left.\mathrm{V}_{\mathrm{L}}\right)$ of antibody $\mathrm{D} 1.3$ was linked to MalE through the pentapeptide N-Ala-AspAla-Ala-Ala-C, the other variable domain was in a free form, and both assembled non-covalently to form a heterodimeric Fv fragment (Figure 1). We chose to link the variable domains to MalE by their C-termini to avoid interference of MalE with the binding of HEL: the C-termini of the variable domains are indeed far away from the antigen combining site and are linked to the constant domains in the natural antibody molecule. Furthermore, a previous work had shown that good results could be obtained, in terms of purity and yield, in this configuration (Brégégère et al., 1994). We prepared hybrids comprising non-covalently assembled vari- able fragments $(\mathrm{Fv})$, rather than single-chain $\mathrm{Fv}$ fragments $(\mathrm{scFv})$, to avoid problems arising from the presence of a polypeptide linker joining $\mathrm{V}_{\mathrm{H}}$ and $\mathrm{V}_{\mathrm{L}}$, such as conformational instability and a tendency to oligomerize and aggregate (Raag \& Whitlow, 1995).

We purified the Fv-MalE hybrids to homogeneity from the producing cells, PD28(pVD82), PD28(pVD91), and their mutant derivatives, in three steps: an osmotic shock, to release the content of the periplasm, an affinity chromatography on a column of cross-linked amylose, using MalE as a tag, and an ion-exchange chromatography, which allowed to discard the hybrids bearing an incomplete Fv moiety (Brégégère et al., 1994). We obtained 1-2 mg of pure FvMalE hybrid per liter of culture medium at $A_{600}=1.5$ (i.e., 12 000-25 000 Fv-MalE molecules per PD28 cell). The yield was limited by a leakiness of the cellular external membrane during the preparation of the periplasmic extracts from the bacterial cultures, which caused $30-50 \%$ of the Fv-MalE molecules to be lost before the osmotic shock. We detected no decrease in the reactivity and affinity of the purified hybrids after 4 months of storage at $4{ }^{\circ} \mathrm{C}$. This observation suggested that no significant dissociation of the Fv moiety of Fv-MalE occurred. It was in accordance with the very high constant $\left(K_{\mathrm{a}}=10^{10} \mathrm{M}^{-1}\right)$ reported for the association of $\mathrm{V}_{\mathrm{H}}$ and $\mathrm{V}_{\mathrm{L}}$ in forming the Fv of D1.3 (Berry \& Pierce, 1993).

(B) Reactivity of the Fv-MalE Hybrids. As the purification of the Fv-MalE hybrids included an affinity chromatography on cross-linked amylose, the purified molecules had a functional MalE moiety. As their purification was independent from their properties of binding to HEL, their Fv moiety could be misfolded and inactive. It was therefore necessary to determine which proportion of the purified Fv-MalE hybrids were indeed able to bind HEL. For this titration, we immobilized a monoclonal antibody directed against MalE on a BIAcore sensorchip, at high density $\left(5 \times 10^{10}\right.$ functional binding sites $/ \mathrm{mm}^{2}$ ), to tightly capture the circulating Fv-MalE hybrids. We then saturated the captured FvMalE hybrids with HEL. The procedure used to calculate the Fv-MalE reactivity toward HEL is detailed in Materials and Methods and illustrated in Figure 2.

It was important to choose a monoclonal antibody that bound MalE stably. We found that this was the case for mAb56.5. We determined the kinetic parameters of the interaction between the Fv-MalE hybrids and the immobilized mAb56.5 (Figure 2: curve "Fv-MalE alone"). The value of $k_{\text {off }}$, about $3 \times 10^{-5} \mathrm{~s}^{-1}$, showed that the complex was very stable once formed. The proportion of Fv-MalE molecules that were able to bind HEL was between 0.95 and 1.10 in all the cases but one. The titer of the H-D100A mutant could not be determined, most probably because its affinity for HEL was too low. These results showed that the total protein concentration, measured by a Bradford assay, could be considered as the concentration of active sites.

(C) Rationale for the Mutagenesis of Antigen Contact Residues of D1.3. The crystal structure of the complex between D1.3 and HEL suggests the existence of 64 direct contacts and of many indirect contacts bridged by water molecules. To measure the energetic and kinetic contributions of various of these contacts, we substituted the residues of D1.3 that are involved, by site-directed mutagenesis of the Fv-MalE hybrids. We changed at least one residue in each of the six CDRs. Table 1 lists the contacts that are 


\begin{tabular}{|c|c|c|}
\hline mutations & atoms $^{b}$ & potential contacts ${ }^{c}$ \\
\hline \multirow[t]{3}{*}{ H-Y32F } & $\mathrm{O}^{\eta}$ & $\mathrm{K} 116-\mathrm{N}^{\zeta}(3.4 \AA)$ \\
\hline & $\mathrm{O}^{\eta}$ & $\mathrm{H}_{2} \mathrm{O} 246(2.9 \AA)$ \\
\hline & $\mathrm{O}^{\eta}$ & $\mathrm{H}_{2} \mathrm{O} 276(3.1 \AA)$ \\
\hline \multirow[t]{3}{*}{ H-D54A } & $\mathrm{O}^{\delta 2}$ & $\mathrm{~T} 118-\mathrm{C}^{\beta}(3.7 \AA), \mathrm{C}^{\gamma 2}(3.6 \AA)$ \\
\hline & $\mathrm{O}^{\delta 2}$ & $\mathrm{H}_{2} \mathrm{O} 291(2.9 \AA) \rightarrow D 119-\mathrm{N}(2.7 \AA)$ \\
\hline & $\mathrm{C}^{\gamma}$ & $\mathrm{T} 118-\mathrm{C}^{\gamma 2}(4.1 \AA)$ \\
\hline \multirow[t]{7}{*}{$\mathrm{H}-\mathrm{D} 100 \mathrm{~N}^{d}$} & $\mathrm{O}^{\delta 1}$ & $N 27-N^{\delta 2}(3.1 \AA)$ \\
\hline & $\mathrm{O}^{\delta 1}$ & $\mathrm{~S} 24-\mathrm{O}^{\gamma}(2.8 \AA)$ \\
\hline & $\mathrm{O}^{\delta 1}$ & $\mathrm{H}_{2} \mathrm{O} 222(2.9 \AA) \rightarrow \mathrm{N} 27-\mathrm{O}^{\delta 1}(2.8 \AA)$ \\
\hline & $\mathrm{O}^{\delta 2}$ & $S 24-N(2.9 \AA)$ \\
\hline & $\mathrm{O}^{\delta 2}$ & $\mathrm{G} 22-\mathrm{O}(3.0 \AA)$ \\
\hline & $\mathrm{O}^{\delta 2}$ & $\mathrm{Y} 23-\mathrm{C}^{\alpha}(3.5 \AA ̊)$ \\
\hline & $\mathrm{O}^{\delta 2}$ & $\begin{array}{l}\mathrm{H}_{2} \mathrm{O} 150(3.0 \AA) \rightarrow \mathrm{H}_{2} \mathrm{O} 155 \\
\quad(2.9 \AA) \rightarrow R 21-\mathrm{O}(2.8 \AA)\end{array}$ \\
\hline \multirow[t]{3}{*}{ H-Y101F } & $\mathrm{O}^{\eta}$ & $Q 121-N(3.0 \AA), C^{\beta}(3.2 \AA), C^{\delta}(3.5 \AA)$ \\
\hline & $\mathrm{O}^{\eta}$ & $\mathrm{D} 119-\mathrm{O}^{\delta 1}(2.7 \AA), \mathrm{C}^{\gamma}(3.7 \AA)$ \\
\hline & $\mathrm{O}^{\eta}$ & V120-N $(3.2 \AA)$ \\
\hline \multirow[t]{3}{*}{ H-R $102 \mathrm{~K}$} & $\mathrm{~N}^{\eta 1}$ & $G 22-O(2.8 \AA)$ \\
\hline & $\mathrm{N}^{\eta 1}$ & L-Y $49-C^{\epsilon 2}(3.7 \AA)$ \\
\hline & $\mathrm{C}^{\zeta}$ & L-Y49- $C^{\delta 2}(4.0 \AA), C^{\epsilon 2}(3.6 \AA), C^{\zeta}(3.9 \AA)$ \\
\hline \multirow[t]{4}{*}{ H-R102M } & $\mathbf{N}^{\eta 1}$ & $G 22-O(2.8 \AA)$ \\
\hline & $\mathbf{N}^{\eta 1}$ & L-Y $49-C^{\epsilon 2}(3.7 \AA)$ \\
\hline & $\mathrm{C}^{\zeta}$ & L-Y49-C $C^{\delta 2}(4.0 \AA), C^{\epsilon 2}(3.6 \AA), C^{\xi}(3.9 \AA)$ \\
\hline & $\mathrm{C}^{\delta}$ & L-Y49-C $\gamma(3.8 \AA), C^{\delta 2}(3.8 \AA)$ \\
\hline \multirow[t]{2}{*}{ L-Y32F } & $\mathrm{O}^{\eta}$ & $\mathrm{H}_{2} \mathrm{Ol} 13(2.8 \AA) \rightarrow D 18-\mathrm{O}^{\delta 1}(3.0 \AA)$ \\
\hline & $\mathrm{O}^{\eta}$ & $\mathrm{H}_{2} \mathrm{O} 152(2.9 \AA) \rightarrow \mathrm{S} 24-\mathrm{O}^{\gamma}(2.8 \AA)$ \\
\hline \multirow[t]{3}{*}{ L-Y50F } & $\mathrm{O}^{\eta}$ & $\mathrm{D} 18-\mathrm{O}^{\delta 1}(3.2 \AA), \mathrm{O}^{\delta 2}(2.7 \AA), \mathrm{C}^{\gamma}(3.4 \AA)$ \\
\hline & $\mathrm{O}^{\eta}$ & $\begin{array}{l}\mathrm{H}_{2} \mathrm{O} \mathrm{O} 74(3.1 \AA) \rightarrow \mathrm{H}_{2} \mathrm{O} 171 \\
\quad(2.9 \AA) \rightarrow L 129-\mathrm{Oxt}(2.8 \AA)\end{array}$ \\
\hline & $\mathrm{O}^{\eta}$ & $\mathrm{H}_{2} \mathrm{O} 173(3.3 \AA)$ \\
\hline \multirow[t]{8}{*}{ L-W92A } & $\mathrm{C}^{\epsilon 3}$ & $\mathrm{Q} 121-\mathrm{C}^{\delta}(3.8 \AA), \mathrm{N}^{\epsilon 2}(3.8 \AA)$ \\
\hline & $\mathrm{C}^{\xi 2}$ & $\mathrm{R} 125-\mathrm{C}^{\gamma}(3.9 \AA), \mathrm{C}^{\delta}(4.0 \AA)$ \\
\hline & $\mathrm{C}^{\zeta 2}$ & $\mathrm{H}_{2} \mathrm{O} 282(3.6 \AA)$ \\
\hline & $C^{\xi 3}$ & $\mathrm{Q} 121-\mathrm{C}^{\delta}(4.0 \AA ̊)$ \\
\hline & $\mathrm{C}^{\xi 3}$ & $\mathrm{I} 124-\mathrm{C}^{\delta 1}(4.1 \AA)$ \\
\hline & $\mathrm{C}^{\eta 2}$ & $\mathrm{R} 125-\mathrm{C}^{\gamma}(3.8 \AA)$ \\
\hline & $\mathrm{C}^{\eta 2}$ & $\mathrm{H}_{2} \mathrm{O} 282(3.6 \AA)$ \\
\hline & $\mathrm{C}^{\eta 2}$ & $\mathrm{H}_{2} \mathrm{O} 384(3.7 \AA ̊)$ \\
\hline
\end{tabular}

\begin{abstract}
${ }^{a}$ Atomic coordinates of the complex between HEL and the Fv fragment of antibody D1.3 were obtained from the Protein Data Bank (reference $1 \mathrm{VFB}$ ). Residues in the $\mathrm{V}_{\mathrm{H}}$ domain are prefixed by $\mathrm{H}$-, and those in the $\mathrm{V}_{\mathrm{L}}$ domain by L-. The HEL residues are not prefixed. ${ }^{b}$ Only the atoms of the D1.3 residues that have been modified or deleted by the point mutation are indicated. ${ }^{c}$ Hydrogen bonds (italicized characters) and Van der Waals contacts (plain characters) with HEL atoms, $\mathrm{H}_{2} \mathrm{O}$ molecules, and atoms from the opposite variable domain that are involved in the $\mathrm{V}_{\mathrm{H}}:: \mathrm{V}_{\mathrm{L}}$ interface. When contacts between D1.3 and HEL are mediated by water molecules, the chain of contacts is indicated by $(\rightarrow)$. Contact distances are indicated in parentheses. Maximum contact distances are as follows (in $\AA$ ): $\mathrm{C}-\mathrm{C}, 4.1 ; \mathrm{C}-\mathrm{N}$, 3.8; $\mathrm{C}-\mathrm{O}, 3.7 ; \mathrm{O}-\mathrm{O}, 3.3 ; \mathrm{O}-\mathrm{N}, 3.4 ; \mathrm{N}-\mathrm{N}, 3.4$. A hydrogen bond is considered to be formed when the distance between the proton donor and the proton acceptor is less than the distances listed above, and the diedral angle between the proton acceptor, the proton, and the proton donor is greater than $120^{\circ} .{ }^{d}$ Mutation H-D100N alters either atom $\mathrm{O}^{\delta 1}$ or $\mathrm{O}^{\delta 2}$ but not both.
\end{abstract}

modified by each mutation, according to the structure. We replaced tyrosines (H-Tyr32, H-Tyr101, L-Tyr32, and LTyr50) by phenylalanines to analyze the role of their hydroxyl group. We changed L-Trp92 into an alanine to study the role of its bulky aromatic cycle. We replaced $\mathrm{H}-\mathrm{Asp} 54$ by an alanine, and H-Asp100 both by an alanine and the more conservative asparagine, to study the role of the carboxyl group of the side chain. Finally, we substituted the long basic side chain of H-Arg 102 both by that of a methionine and of a lysine, to alter the contacts of this residue with HEL but not its contribution to the $\mathrm{V}_{\mathrm{H}}:: \mathrm{V}_{\mathrm{L}}$ interface.

To study the effect of mutations involving residues from $\mathrm{V}_{\mathrm{H}}$, we chose hybrid $\mathrm{V}_{\mathrm{L}}:: \mathrm{V}_{\mathrm{H}}-\mathrm{MalE}$, expressed from ph- agemid pVD82. Conversely, to study the effect of mutations involving residues from $\mathrm{V}_{\mathrm{L}}$, we chose the symmetrical hybrid, $\mathrm{V}_{\mathrm{H}}:: \mathrm{V}_{\mathrm{L}}-\mathrm{MalE}$, expressed from $\mathrm{pVD} 91$. Therefore, the mutated variable domain was always fused with MalE.

(D) Kinetic Parameters for the Binding of the Wild-Type and Mutant Fv-MalE to HEL. The fraction of immobilized HEL molecules that was available for Fv-MalE binding, was between $50 \%$ and $60 \%$ at the surface concentration used, as determined from eq 2 (Materials and Methods). We fitted functions comprising one or two exponential terms to the kinetic profiles obtained with the BIAcore apparatus for the interaction between the Fv-MalE hybrids and immobilized HEL. We used function 4 or 8 for the association process and function 3 or 7 for the dissociation process (Materials and Methods). In each case, we evaluated the goodness of fitting according to two criteria: the $\chi^{2}$ parameter and the dispersion of the residual values. Function 8 , which includes two exponential terms, fitted the experimental data of the association process better at high concentrations of Fv-MalE $(C>150 \mathrm{nM})$, while function 4 , which includes only one exponential term, fitted them better at lower concentrations $(C<150 \mathrm{nM})$. Function 7, which comprises two exponential terms, fitted the experimental data of the dissociation process better at all concentrations of Fv-MalE except very low ones $(C<10 \mathrm{nM})$. These observations indicated that the epitope of D1.3 was presented in a heterogeneous way on the immobilized HEL molecules, and that the access of FV-MalE to a significant proportion of these molecules was hindered. These two phenomena have been widely observed, both with the BIAcore and the Fisons IAsys sensor, when protein ligands are covalently immobilized on a carboxymethyl dextran matrix through amide bonds with their free side-chain amine groups. As a result of the immobilization method, the ligand molecules are randomly distributed over and within the matrix, and presented in several different configurations (Borrebaeck et al., 1992; O'Shannessy et al., 1993; Edwards et al., 1995).

When the immobilized molecules are heterogeneous as here, and when a sum of two exponentials is fitted to the association profile, only the larger time constant $k_{\text {obs2 }}$ can be interpreted as an apparent association rate constant, while the smaller one $k_{\mathrm{obs} 1}$ contains no readily interpretable kinetic information (Edwards et al., 1995). We found that the value of the ratio $R_{\text {eq2 }} k_{\text {obs } 2} /\left(R_{\text {eq1 }} k_{\mathrm{obs} 1}+R_{\text {eq } 2} k_{\text {obs } 2}\right)$ was always higher than 0.935 , i.e., the contribution of the slow phase to the initial rate of association was always less than $6.5 \%$ (see eq 9 ). Therefore, to determine the association rate constant $k_{\mathrm{on}}$, we plotted either $k_{\mathrm{obs}}$ (for $C<150 \mathrm{nM}$ ) or $k_{\mathrm{obs} 2}$ (for $C>$ $150 \mathrm{nM}$ ) versus $C$. Equation 5 could be fitted to these combined plots with linear regression coefficients higher than 0.985. As dissociation rate constant $k_{\text {off }}$, we took that of the two partial dissociation rate constants $\left(k_{\text {off } 1}\right.$ and $k_{\text {off2 } 2}$, averaged over the concentrations) which was closer to the $y$-intercept value of the $k_{\mathrm{obs}}$ versus $C$ combined plots (Materials and Methods). The $k_{\text {on }}, k_{\text {off }}$, and $K_{\mathrm{d}}^{\prime}$ values measured at $20^{\circ} \mathrm{C}$ for the wild-type and the mutant Fv-MalE hybrids are given in Table 2. The values of the parameters for the two wildtype hybrids, $\mathrm{V}_{\mathrm{L}}:: \mathrm{V}_{\mathrm{H}}-\mathrm{MalE}$ and $\mathrm{V}_{\mathrm{H}}:: \mathrm{V}_{\mathrm{L}}-$ MalE, were not significantly different. The $k_{\mathrm{on}}$ values for the 10 mutant hybrids differed from the $k_{\text {on }}$ of the wild-type by a factor of less than 3 , whereas their $k_{\text {off }}$ values showed differences as large as 3 orders of magnitude, in the case of mutations H-D100N, H-R102M, and L-W92A. 


\begin{tabular}{|c|c|c|c|c|c|}
\hline mutation & CDR & $k_{\mathrm{on}}\left(10^{-4} \mathrm{M}^{-1} \cdot \mathrm{s}^{-1}\right)$ & $k_{\text {off }}\left(10^{4} \mathrm{~s}^{-1}\right)$ & $K_{\mathrm{d}}^{\prime}(\mathrm{nM})$ & $\Delta G^{\prime}\left(\mathrm{kcal} \cdot \mathrm{mol}^{-1}\right)$ \\
\hline \multicolumn{6}{|l|}{$\mathrm{V}_{\mathrm{L}}:: \mathrm{V}_{\mathrm{H}}-\mathrm{MalE}$} \\
\hline wt & & $7.84 \pm 0.45$ & $6.66 \pm 0.79$ & $8.46 \pm 0.73$ & $10.82 \pm 0.05$ \\
\hline H-Y32F & 1 & $8.26 \pm 0.08$ & $9.96 \pm 0.34$ & $12.1 \pm 0.5$ & $10.61 \pm 0.03$ \\
\hline H-D54A & 2 & $11.8 \pm 0.7$ & $186 \pm 12$ & $163 \pm 22$ & $9.11 \pm 0.07$ \\
\hline H-D100N & 3 & $20.7 \pm 3.6$ & $10300 \pm 1000$ & $5000 \pm 400$ & $7.10 \pm 0.05$ \\
\hline H-D100A & 3 & $\mathrm{~nm}^{c}$ & $\mathrm{~nm}$ & $\mathrm{~nm}$ & $\mathrm{~nm}$ \\
\hline $\mathrm{H}-\mathrm{Y} 101 \mathrm{~F}$ & 3 & $9.68 \pm 0.31$ & $622 \pm 27$ & $643 \pm 23$ & $8.30 \pm 0.02$ \\
\hline H-R $102 \mathrm{~K}$ & 3 & $13.5 \pm 0.7$ & $169 \pm 5$ & $126 \pm 6$ & $9.25 \pm 0.03$ \\
\hline H-R102M & 3 & $10.6 \pm 1.1$ & $2240 \pm 120$ & $2160 \pm 80$ & $7.59 \pm 0.02$ \\
\hline \multicolumn{6}{|l|}{$\mathrm{V}_{\mathrm{H}}:: \mathrm{V}_{\mathrm{L}}-\mathrm{MalE}$} \\
\hline wt & & $8.66 \pm 1.02$ & $7.51 \pm 0.29$ & $8.96 \pm 0.91$ & $10.79 \pm 0.06$ \\
\hline L-Y32F & 1 & $8.24 \pm 0.16$ & $294 \pm 6$ & $357 \pm 12$ & $8.64 \pm 0.02$ \\
\hline L-Y50F & 2 & $8.76 \pm 0.37$ & $17.7 \pm 0.5$ & $20.3 \pm 0.3$ & $10.31 \pm 0.01$ \\
\hline L-W92A & 3 & $11.1 \pm 0.6$ & $5700 \pm 400$ & $5120 \pm 590$ & $7.09 \pm 0.07$ \\
\hline
\end{tabular}

${ }^{a}$ The determination of $k_{\mathrm{on}}, k_{\mathrm{off}}$, and $K_{\mathrm{d}}^{\prime}$ from BIAcore experimental data is described in Materials and Methods. $\Delta G^{\prime}$ values were calculated as $-R T \ln K_{\mathrm{d}}^{\prime}$, where $R$ is the gas constant and $T$ the absolute temperature. The mean value and associated standard error of 2 or more independent determinations are given. ${ }^{b} K_{\mathrm{d}}^{\prime}$ is the equilibrium dissociation constant between Fv-MalE and HEL measured with the BIAcore apparatus at the heterogeneous interface between the liquid phase and the sensorchip. There is no simple relationship between this constant and the constant $\mathrm{K}_{\mathrm{d}}$ that would be measured in a homogeneous solution. ${ }^{c} \mathrm{~nm}$, not measurable.

\section{DISCUSSION}

(A) MalE as an Affinity Handle for the Purification and Titration of $F v$ Fragments. We used Fv-MalE hybrids between the variable fragment $(\mathrm{Fv})$ of antibody D1.3 and MalE as such, without cleavage by site-specific proteases, to analyze its interaction with HEL. The yields of the purified Fv-MalE hybrids $(1-2 \mathrm{mg} / \mathrm{L}$ of culture medium at $A_{600}=1.5$ ) were relatively low when compared to those of hybrids between Fv fragments and calmodulin (Neri et al., 1995) but were sufficient for their characterization with the BIAcore apparatus. Various strategies described for free Fv or $\mathrm{scFv}$ could be used to improve the periplasmic expression of Fv-MalE (Knappik et al., 1993; Ayala et al., 1995; Knappik \& Pluckthun, 1995).

The Fv-MalE hybrids were purified by methods which did not involve the use of affinity chromatography on immobilized HEL. It was therefore necessary to measure the proportion of Fv-MalE molecules that were able to bind HEL before determining the kinetic parameters of the interaction. We devised a titration method in which Fv-MalE is first captured by a monoclonal antibody directed against MalE, then saturated with HEL (Figure 2). We found that the Fv moieties of the wild-type and mutant Fv-MalE hybrids were fully functional in all but one case (H-D100A). These results showed that the purification protocol described here is a valid alternative to antigen affinity chromatography, with the distinct advantage that there is no impediment for the purification of low-affinity mutants. No similar titration method has yet been developed for antibody fragments fused to other protein or peptide tags, although the question of the functionality of tagged-antibody fragments is important for many applications. However, a method described for the quantitation of hexa-histidine-tagged recombinant proteins (O'Shannessy et al., 1995) as well as the glutathione $S$-transferase kit for fusion capture (Pharmacia Biosensor) could be used to this avail.

(B) Validation of Fv-MalE for Mutagenesis Studies. It was crucial to determine whether the MalE moiety of the Fv-MalE hybrids altered the interaction between their Fv moiety and HEL. We first showed that there was no significant difference between the binding properties of the two symmetrical wild-type hybrids, $\mathrm{V}_{\mathrm{L}}:: \mathrm{V}_{\mathrm{H}}-\mathrm{MalE}$ and $\mathrm{V}_{\mathrm{H}}$ :
Table 3: Effect of Mutations on the Free Energy of Interaction between HEL and the Fv Fragment of Antibody D1.3 When Fused or Not with MalE

\begin{tabular}{lccl}
\hline mutation & CDR & $\begin{array}{c}\Delta \Delta G(\mathrm{Fv}-\mathrm{MalE})^{a} \\
\left(\mathrm{kcal} \cdot \mathrm{mol}^{-1}\right)\end{array}$ & $\begin{array}{c}\Delta \Delta G(\text { free Fv })^{b} \\
\left(\mathrm{kcal} \cdot \mathrm{mol}^{-1}\right)\end{array}$ \\
\hline H-Y32F & 1 & $0.21 \pm 0.06$ & $0.41 \pm 0.25$ \\
H-D54A & 2 & $1.71 \pm 0.09$ & $1.94 \pm 0.18$ \\
H-D100N & 3 & $3.72 \pm 0.07$ & $\mathrm{na}^{c}$ \\
H-Y101F & 3 & $2.52 \pm 0.05$ & $2.33 \pm 0.23$ \\
H-R102K & 3 & $1.58 \pm 0.06$ & na \\
H-R102M & 3 & $3.23 \pm 0.05$ & na \\
L-Y32F & 1 & $2.15 \pm 0.06$ & $1.97 \pm 0.19$ \\
L-Y50F & 2 & $0.48 \pm 0.06$ & $0.81 \pm 0.25$ \\
L-W92A & 3 & $3.70 \pm 0.09$ & $1.70 \pm 0.21$ \\
\hline
\end{tabular}

${ }^{a} \Delta \Delta G$ values for Fv-MalE were determined as $\Delta G_{\mathrm{wt}}^{\prime}-\Delta G_{\mathrm{mut}}^{\prime}$, using the BIAcore data shown in Table 2. For each mutation, the standard error on $\Delta \Delta G$ was calculated from the standard errors on $\Delta G_{\mathrm{wt}}^{\prime}$ and $\Delta G_{\text {mut }}^{\prime}$ using the formula $(\operatorname{SE}(\Delta \Delta G))^{2}=\left(\operatorname{SE}\left(\Delta G^{\prime}{ }_{\mathrm{wt}}\right)\right)^{2}+$ $\left(\operatorname{SE}\left(\Delta G_{\text {mut }}^{\prime}\right)\right)^{2}$. wt, wild type; mut, mutant; SE, standard error. ${ }^{b} \Delta \Delta G$ values for the free Fv were determined as $\Delta G_{\mathrm{wt}}-\Delta G_{\mathrm{mut}}$, where $\Delta G$ $=-R T \ln K_{\mathrm{d}}$, using the $K_{\mathrm{d}}$ values previously measured at $20{ }^{\circ} \mathrm{C}$ by fluorescence quench titration (Hawkins et al., 1993). The standard errors on the $\Delta G$ values were deduced from the standard errors reported for the $K_{\mathrm{d}}$ values, using confidence intervals of $95 \%$. For instance, $\Delta G_{\mathrm{wt}}=11.30 \pm 0.16 \mathrm{kcal} \cdot \mathrm{mol}^{-1}$. The standard error on $\Delta \Delta G$ was calculated as described in $a .{ }^{c}$ Not available.

$: \mathrm{V}_{\mathrm{L}}-\mathrm{MalE}$, when measured with the BIAcore apparatus (table 2). This identity suggested that either MalE had no effect on the interaction between the Fv and the immobilized HEL, or that the effect of MalE did not depend on the variable domain, $\mathrm{V}_{\mathrm{H}}$ or $\mathrm{V}_{\mathrm{L}}$, which was linked to it. Similar results had previously been obtained using a competition ELISA as binding assay (Brégégère et al., 1994).

To study more thoroughly the potential effect of MalE on the interaction between the Fv and HEL, we used mutations of residues from all six CDR loops of D1.3 as local conformational probes. The effects of these mutations on the binding of Fv-MalE to HEL were determined with the BIAcore apparatus, and compared with those observed for the free Fv (i.e., not fused with MalE) by fluorescence quench titration (Hawkins et al., 1993) (Table 3). For each mutation, a difference in the $\Delta \Delta G$ s measured in the two contexts should only reflect the differences in the way in which FvMalE and free Fv contact HEL. Theoretical reasons and experimental results indeed indicate that $\Delta \Delta G$ s measured 
by methods in solution (such as fluorescence quench titration) are identical to those measured with the BIAcore. The free energies of translation and rotation that are lost when one partner of the interaction is immobilized on a chip do not depend on the presence of a mutation in the soluble partner. Furthermore, in the case of the association between the human growth hormone and its receptor, the values of $\Delta \Delta G$ corresponding to point mutations in the growth hormone are identical whether they are measured in solution by RIA or with the BIAcore (Cunningham \& Wells, 1993). The functional method of comparison between two related macromolecules that we used here is an alternative to structural approaches such as X-ray crystallography, with the advantage that it is less time-consuming and that its results could be more biologically meaningful.

The effects of the probe mutations on the free energy of the interaction between HEL and either FV-MalE or free FV were very close, within experimental errors, in all cases except L-W92A. A site-specific effect of MalE on the L-CDR3 loop of Fv-MalE cannot be ruled out in this latter case. However, the 500-fold effect that we measured for L-W92A in the Fv-MalE context, is more consistent with other experimental data than the 20-fold effect reported in the free Fv context (Hawkins et al., 1993). Another mutation at the same position, L-W92D, indeed decreases the affinity of the free Fv for HEL by 3 orders of magnitude (Ysern et al., 1994). We concluded that the Fv fragment of D1.3 formed the same contacts with HEL, both qualitatively and quantitatively, whether it was fused with MalE or not. Our results therefore validate the use of Fv-MalE hybrids for structural and functional studies on the interactions between antibody and antigen. For many other applications of the Fv-MalE hybrids, it may also be unnecessary to remove the MalE moiety. This possibility is especially interesting, as the site-specific removal of protein tags after purification often involves costly, time-consuming, and unreliable enzymatic cleavage methods and is generally considered as a serious bottleneck.

(C) Analysis of the Interaction between D1.3 and HEL Using Site-Directed Mutagenesis. Mutagenesis studies have shown that only a subset of the CDRs side chains play an important part in the energy of interaction between antibody hu4D5-5 and receptor p185 ${ }^{\text {HER2 }}$ (Kelley \& O'Connell, 1993). Similar hot spots of binding energy were observed at the interface between the human growth hormone and its receptor (Cunningham \& Wells, 1993; Clackson \& Wells, 1995) and at that between barnase and its intracellular inhibitor, barstar (Schreiber \& Fersht, 1993, 1995). To understand more precisely the energetic and kinetic contributions of the contacts defined by structural methods in the interaction between D1.3 and HEL, we constructed 10 different point mutations in hybrid Fv-MalE and measured their effects on the kinetic parameters of binding to HEL, using the BIAcore apparatus. Six of these mutations were also used for the comparative study described in the previous paragraph. The four other ones changed two residues from the H-CDR3 loop, H-Asp100 and H-Arg102, whose energetic importance failed to be determined in previous mutagenesis studies (Hawkins et al., 1993).

(D) Effects of the CDR Mutations on $k_{\text {on }}$. The point mutations introduced in Fv-MalE changed the $k_{\text {on }}$ for HEL at most 3-fold (Table 2). Similar results have been observed for other antibodies, human growth hormone and its receptor, and the barnase-barstar complex (Kelley \& O'Connell, 1993; Cunningham \& Wells, 1993; Clackson \& Wells, 1995; Schreiber \& Fersht, 1993, 1995). The small variations of $k_{\text {on }}$ suggested in particular that the mutations did not modify the backbone structure of the CDRs of D1.3. We expected that these mutations would not induce gross structural changes, as we substituted the side chains of D1.3 by smaller side chains, we did not introduce any proline, and none of the residues concerned was involved in an intradomain stabilizing hydrogen bond, according to the X-ray structure.

Productive encounters between antibody and antigen involves successively long-range attraction forces, which are of hydrophobic or electrostatic nature, then short-range interactions, which result in the establishment of the actual hydrogen or Van der Waals bonds present in the complex (Kozack \& Subramanian, 1993; Van Oss, 1995). Therefore, the small effects of the mutations on $k_{\text {on }}$ indicated that the close-range interactions, which should depend on the exact nature of the CDR side chains, played a minor part in the process of association between D1.3 and HEL. They also showed that the point mutations did not change significantly the hydrophobicity or overall electrostatic charge of the D1.3 CDRs, when introduced individually. We found that mutations which resulted in the loss of either positive (H-R102M) or negative (H-D54A and H-D100N) charges in the paratope, did not induce greater variations of $k_{\mathrm{on}}$. This observation was surprising since there are only seven charged residues in the CDRs of D1.3 (four positively and three negatively charged). It suggests either that electrostatic interactions do not play a fundamental part in the initial encounter between D1.3 and HEL, or more probably that residues belonging to the $\beta$-sheet framework contribute significantly to the overall electrostatic charge of the paratope.

We observed that the mutations either increased $k_{\text {on }}$ or left it unchanged. Similar increases have been reported for multiple mutants of D1.3 (Ito et al., 1995). However, the variations are generally negative in other systems and are much greater for double than for single mutations (Kelley \& O'Connell, 1993; Schreiber \& Fersht, 1995). Our results suggest that the distribution of electrostatic charges throughout the HEL-combining site of D1.3 is not optimized for long-range interactions.

(E) Effects of the CDR Mutations on $k_{\text {off. }}$ The mutations in Fv-MalE always increased $k_{\text {off }}$, up to 1000 times (Table 2 ). These increases showed that the dissociation process was very sensitive to the exact nature of the interacting side chains and suggested that it relied primarily on the disruption of short-range contacts. The increases of $k_{\text {off }}$ due to the mutations in H-CDR3 (D100N, R102M, and Y101F) and L-CDR3 (W92A) were 100-fold or more, while for mutations in L-CDR1 (Y32F) and H-CDR2 (D54A) they were 10100-fold, and finally for mutations in L-CDR2 (Y50F) and H-CDR1 (Y32F), they were less than 10-fold. H-CDR3 and L-CDR3 of D1.3 are contiguous and form the center of the HEL-combining site. Our results showed that their residues played a predominant part in the energy of the interaction between D1.3 and HEL, with respect to the residues of the more peripheral zones of the paratope. Similar observations have been reported for the complexes between antibody hu4D5-5 and receptor p185 ${ }^{\mathrm{HER} 2}$ and between the human growth hormone and its receptor (Kelley \& O'Connell, 1993; Cunningham \& Wells, 1993; Clackson \& Wells, 1995). Furthermore, we found that some charged residues of the 
CDRs of D1.3 (H-Asp100, H-Arg102, and, to a lesser extent, H-Asp54) were at least as important for the interaction as their aromatic residues. Thus, the aromatic residues do not necessarily play a predominant part in the energy of interaction between antibody and antigen, even though tyrosines are very frequent among the antigen-binding residues of antibodies (Mian et al., 1991). However, in the case of the tyrosine residues (H-Tyr32, H-Tyr101, L-Tyr32, and L-Tyr50), we only investigated the role of the hydroxyl group by mutations into phenylalanines and not that of the aromatic cycle itself, which can be crucial (our L-W92A mutation; Kelley \& O'Connell, 1993; Tsumoto et al., 1995).

(F) Comparison of the Mutagenesis Results with Crystallographic Data. The crystal structure of the complex between the Fv fragment of D1.3 and HEL allowed us to predict the number and nature of the bonds that would be altered by mutations (Table 1). We compared these predictions with the measured effects for each of the 10 mutations that we introduced into D1.3 (Table 3). We found that mutations which deleted one or more hydrogen bonds between D1.3 and HEL induced a high $\Delta \Delta G(>1.5$ $\left.\mathrm{kcal} \cdot \mathrm{mol}^{-1}\right)$, whether these bonds were direct, as in the case of mutations H-D100N, H-Y101F, H-R102K, and H-R102M, or mediated by one water molecule, as in the case of mutations H-D54A, H-D100N, and L-Y32F. The disruption of contacts between proton donors and proton acceptors which did not meet the angle conditions for the formation of a hydrogen bond had virtually no energetic consequence, as in the case of mutations H-Y32F and L-Y50F. The high $\Delta \Delta G\left(3.7 \mathrm{kcal} \cdot \mathrm{mol}^{-1}\right)$ induced by mutation L-W92A showed that apolar Van der Waals contacts between the aromatic ring of L-Trp92 and HEL were also energetically important.

We attempted to evaluate the energy of several types of bonds between D1.3 and HEL from our data, by assuming that the only effect of the point mutations was to alter the contacts listed in Table 1, and by using the fact that the energy of a $\mathrm{C} \cdot \mathrm{O}$ contact is negligible when the distance between the two atoms is above $2.7 \AA$ (Derewenda et al., 1995). In these conditions, the $\Delta \Delta G$ induced by mutation L-W92A $\left(3.7 \mathrm{kcal} \cdot \mathrm{mol}^{-1}\right)$ would result from the disruption of seven $\mathrm{C} \cdot \mathrm{C}$ or $\mathrm{C} \cdot \mathrm{N}$ contacts between the aromatic ring of L-Trp92 and HEL, whose average energy would be 0.5 $\mathrm{kcal} \cdot \mathrm{mol}^{-1}$ each, as previously reported (Ysern et al., 1994). The $\Delta \Delta G$ induced by $\mathrm{H}-\mathrm{Y} 101 \mathrm{~F}\left(2.5 \mathrm{kcal} \cdot \mathrm{mol}^{-1}\right)$ would result from the disruption of a direct hydrogen bond, whose energy would fall within the range for this type of bond (Fersht \& Serrano, 1993). The $\Delta \Delta G$ s induced by L-Y32F and H-D54A (2.1 and $1.7 \mathrm{kcal} \cdot \mathrm{mol}^{-1}$ ) would result primarily from the disruption of a water-mediated hydrogen bond, whose energy would be $1-2 \mathrm{kcal} \cdot \mathrm{mol}^{-1}$. The energy for this type of indirect bond has never been estimated before. Our estimations are consistent with the $\Delta \Delta G$ induced by H-D100N (3.7 $\left.\mathrm{kcal} \cdot \mathrm{mol}^{-1}\right)$, which disrupts both a direct and a watermediated hydrogen bond.

Although the mutations that we constructed did not alter all the residues of D1.3 that contact HEL, the sum of their $\Delta \Delta G \mathrm{~s}$, measured with the BIAcore apparatus $\left(16.0 \mathrm{kcal} \cdot \mathrm{mol}^{-1}\right.$ using mutation $\mathrm{H}-\mathrm{R} 102 \mathrm{~K}$, or $17.6 \mathrm{kcal} \cdot \mathrm{mol}^{-1}$ using mutation H-R102M; Table 3) was higher than the $\Delta G^{\circ}$ of the interaction between D1.3 and HEL, measured by fluorimetry or microcalorimetry, 11.3 and $11.5 \mathrm{kcal} \cdot \mathrm{mol}^{-1}$, respectively (Hawkins et al., 1993; Bhat et al., 1994). The bond energies calculated above should therefore be taken as overestimations of the real values. A similar disparity between the sum of the $\Delta \Delta G$ s corresponding to individual mutations and the $\Delta G^{\circ}$ was found in most cases when the structural interface between two proteins has been analyzed by a mutational approach, whatever the method of measurement used (Kelley \& O'Connell, 1993; Schreiber \& Fersht, 1993; Clackson \& Wells, 1995).

There are two theoretical reasons for which $\Delta \Delta G$ is an overestimation of $\Delta \Delta G_{\text {bind }}$, the incremental binding energy which is brought by the mutated side chain (Horovitz, 1987; Fersht, 1988). First, the association of D1.3 and HEL exchanges non-covalent interactions that the free D1.3 and the free HEL make with the solvent for interactions that they make together. A mutation in D1.3 will generally change the interactions that both the free D1.3 and its complex with HEL make with the solvent. Second, a mutation in the antigen-combining site of D1.3 can modify the conformation and the physicochemical environment of neighboring residues that are also involved in the interaction with HEL. Crystallographic data have brought structural evidence for the above effects in the case of the association between the Fv fragment of antibody D1.3 and HEL. A comparison of the crystal structures of the wild type complex and of a mutant complex in which L-Trp92 is changed into Asp, has indeed shown that the mutation modifies the structure of several neighbouring side chains and that two additional water molecules in the mutant replace the aromatic part of the Trp side chain in the wild type (Ysern et al., 1994). Preliminary results concerning five other mutations have also shown that they cause modifications in the hydration of the interface (Braden et al., 1995). Although not yet demonstrated in the case of D1.3, the general finding that multiple mutations within a protein can have nonadditive effects on its interaction with a ligand has also brought functional evidence that a mutation can modify the properties of the neighbouring residues (Carter et al., 1984; Ackers \& Smith, 1985; Wells, 1990; Mildvan et al., 1992).

A precise understanding of the effects of mutations on the interaction between antibody D1.3 and HEL and of the energetic importance of the water-mediated contacts awaits additional data, such as the refined crystal structures of several more complexes between D1.3 mutants and HEL, and the identification of the functional interactions between side chains of the CDRs through the thorough study of the non-additivity of multiple mutations. These matters are currently being looked into.

\section{NOTE ADDED DURING THE REVISION OF THE MANUSCRIPT}

After submission of this paper and while we were revising the manuscript, we became aware that another group had simultaneously studied the interface between antibody D1.3 and HEL, through an alanine scan approach (Dall' Acqua et al., 1996). Both studies agree about the major contribution of four residues in the strength of the interaction (H-Asp100, L-Trp92, H-Tyr101, and L-Tyr32). Additionally, we showed that H-Arg102, which these authors did not test, also played a major functional part. Surprisingly, Dall' Acqua et al. report values of $\Delta \Delta G$ for mutations $\mathrm{H}-\mathrm{D} 54 \mathrm{~A}$ and $\mathrm{H}-\mathrm{Y} 101 \mathrm{~F}$ which are significantly lower than those that we and G. Winter's group have found (this work; Hawkins et al., 1993; see Table 3). Furthermore, they report lower $\Delta \Delta G$ s for the nonconservative mutations H-D100A and L-Y32A than those 
that we and others have found for the more conservative mutations H-D100N and L-Y32F (Table 3). As suggested by Dall'Acqua et al. (1996), the disparities could arise from the fact that they used the single-chain $\mathrm{scFv}$ of D1.3, in which an artificial peptide links the $\mathrm{V}_{\mathrm{H}}$ and $\mathrm{V}_{\mathrm{L}}$ domains. This linker was not present in either the Fv-MalE hybrids or the free Fv that we and G. Winter's group used. The disparities could also come from the different methods in which the $K_{\mathrm{d}}^{\prime}$ values were determined from the BIAcore data. Dall'Acqua et al. deduced the value of $K^{\prime}{ }_{\mathrm{d}}$ from the SPR response at the steady-state, whereas we measured $k_{\text {off }}$ and $k_{\text {on }}$ and deduced $K_{\mathrm{d}}^{\prime}$ from their ratio. Moreover, they considered only one phase of association whereas we took into account the heterogeneity in the population of immobilized lysozyme.

\section{ACKNOWLEDGMENT}

We thank Dr. Jean-Michel Betton and Dr. Maurice Hofnung for the gift of monoclonal antibody 56-5 and Dr. Malcolm Buckle for assistance with the BIAcore apparatus.

\section{REFERENCES}

Ackers, G. K., \& Smith, F. R. (1985) Annu. Rev. Biochem. 54, 597-629.

Ayala, M., Balint, R. F., Fernandez de Cossio, M. E., Canaanhaden, L., Larrick, J. W., \& Gavilondo, J. V. (1995) BioTechniques 18, 832-841.

Bedouelle, H., \& Duplay, P. (1988) Eur. J. Biochem. 171, 541549.

Berry, M. J., \& Pierce, J. J. (1993) J. Chromatogr. 629, 161-168.

Bhat, T. N., Bentley, G. A., Boulot, G., Greene, M. I., Tello, D., Dall'Acqua, W., Souchon, H., Schwarz, F. P., Mariuzza, R. A., \& Poljak, R. J. (1994) Proc. Natl. Acad. Sci. U.S.A. 91, 10891093.

Borrebaeck, C. A. K., Malmborg, A-C., Furebring, C., Michaelsson, A., Ward, S., Danielsson, L., \& Ohlin, M. (1992) Biotechnology 10, 697-398.

Braden, B. C., \& Poljak, R. J. (1995) FASEB J. 9, 9-16.

Braden, B. C., Fields, B. A., \& Poljak, R. J. (1995) J. Mol. Recognit. 8, 317-325.

Brégégère, F., Schwartz, J., \& Bedouelle, H. (1994) Protein Eng. 7, 271-280.

Brinkmann, U., \& Pastan, I. (1994) Biochim. Biophys. Acta 1198, 27-45.

Carter, P. J., Winter, G., Wilkinson, A., \& Fersht, A. R. (1984) Cell 38, 835-840.

Clackson, T., \& Wells, J. A. (1995) Science 267, 383-386.

Cunningham, B. C., \& Wells, J. A. (1993) J. Mol. Biol. 234, 554563.

Dall'Acqua, W., Goldman, E. R., Eisenstein, E., \& Mariuzza, R. A. (1996) Biochemistry 35, 9667-9676.

Davies, D. R., \& Cohen, G. H. (1996) Proc. Natl. Acad. Sci. U.S.A. 93, 7-12.

Derewenda, Z. S., Lee, L., \& Derewenda, U. (1995) J. Mol. Biol. 252, 248-262.

Dübel, S., Breitling, F., Kontermann, R., Schmidt, T., Skerra, A., \& Little, M. (1995) J. Immunol. Meth. 178, 201-209.
Duplay, P., Szmelcman, S., Bedouelle, H., \& Hofnung, M. (1987) J. Mol. Biol. 194, 663-673.

Edwards, P. R., Gill, A., Pollard-Knight, D. V., Hoare, M., Buckle, P. E., Lowe, P.A., \& Leatherbarrow, R. J. (1995) Anal. Biochem. 231, 210-217.

Fersht, A. R. (1988) Biochemistry 27, 1577-1580.

Fersht, A. R., \& Serrano, L. (1993) Curr. Opin. Struct. Biol. 3, 75-83.

Goshorn, S. C., Svensson, H. P., Kerr, D. E., Somerville, J. E., Senter, P. D., \& Fell, H. P. (1993) Cancer Res. 53, 2123-2127.

Hawkins, R. E., Russell, S. J., Baier, M., \& Winter, G. (1993) J. Mol. Biol. 234, 958-964.

Horovitz, A. (1987) J. Mol. Biol. 196, 733-735.

Ito, W., Iba, Y., \& Kurosawa, Y. (1993) J. Biol. Chem. 268, 1663916647.

Ito, W., Hasui, H., \& Kurosawa, Y. (1995) J. Mol. Biol. 248, 729732.

Janin, J., \& Chothia, C. (1990) J. Biol. Chem. 265, 16027-16030.

Kelley, R. F., \& O'Connell, M. P. (1993) Biochemistry 32, 68286835.

Knappik, A., \& Plückthun, A. (1995) Protein Eng. 8, 81-89.

Knappik, A., Krebber, C., \& Plückthun, A. (1993) Biotechnology $11,77-83$.

Kozack, R. E., \& Subramanian, S. (1993) Protein Sci. 2, 915926.

Kunkel, T. A., Roberts, J. D., \& Zakour, R. A. (1987) Methods Enzymol. 154, 367-382.

Malmqvist, M. (1993) Curr. Opin. Immunol. 5, 282-286.

Mead, D. A., Szczesna-Skorupa, E., \& Kemper, B. (1986) Protein Eng. 1, 67-74.

Mian, I. S., Bradwell, A. R., \& Olson, A. J. (1991) J. Mol. Biol. 217, 133-151.

Mildvan, A. S., Weber, D. J., \& Kuliopulos, A. (1992) Arch. Biochem. Biophys. 294, 327-340.

Neri, D., de Lalla, C., Petrul, H., Neri, P., \& Winter, G. (1995) BioTechnology 13, 373-377.

Neuberger, M. S., Williams, G. T., \& Fox, R. O. (1984) Nature 312, 604-608.

Newton, D. L., Xue, Y., Olson, K. A., Fett, J. W., \& Rybak, S. M. (1996) Biochemistry 35, 545-553.

O'Shannessy, D. J., Brigham-Burke, M., Soneson, K. K., Hensley, P., \& Brooks, I. (1993) Anal. Biochem. 212, 457-468.

O'Shannessy, D. J., O'Donnell, K. C., Martin, J., \& Brigham-Burke, M. (1995) Anal. Biochem. 229, 119-124.

Raag, R., \& Whitlow, M. (1995) FASEB J. 9, 73-80.

Richarme, G. (1982) Biochem. Biophys. Res. Commun. 105, 476481.

Schreiber, G., \& Fersht, A. R. (1993) Biochemistry 32, 5145-5150.

Schreiber, G., \& Fersht, A. R. (1995) J. Mol. Biol. 248, 478-486.

Stenberg, E., Persson, B., Roos, H., \& Urbaniczky, C. (1991) J. Colloid Interface Sci. 143, 513-526.

Tsumoto, K., Ogasahara, K., Ueda, Y., Watanabe, K., Yutani, K., \& Kumagai, I. (1995) J. Biol. Chem. 270, 18551-18557.

Van Oss, C. J. (1995) Mol. Immunol. 32, 199-211.

Webster, D. M., Henry, A. H., \& Rees, A. R. (1994) Curr. Opin. Struct. Biol. 4, 123-129.

Wells, J. A. (1990) Biochemistry 29, 8509-8517.

Wels, W., Harwerth, I.-M., Zwickl, M., Hardman, N., Groner, B., \& Hynes, N. E. (1992) Biotechnology 10, 1128-1132.

Ysern, X., Fields, B. A., Bhat, T. N., Goldbaum, F. A., Dall'Acqua, W., Schwarz, F.P., Poljak, R. J., \& Mariuzza, R. A. (1994) J. Mol. Biol. 238, 496-500.

BI961419Y 